\title{
Lorsque le père ou la mère est malade, comment vont les enfants?
}

\section{Ursula Steiner-König}

Déléguée de la FMH au Comitế de la «Journée des malades»
Cette question ne manque pas de traverser l'esprit du médecin confronté à une grave maladie menaçant la vie d'une mère ou d'un père de famille. Mais on préfèrerait la mettre aussitôt de côté, car la personne malade mobilise déjà suffisamment d'énergie. Et lorsqu'on est soi-même parent d'enfants mineurs, on se sent interpellé et on aurait tendance à s'identifier à la situation. Pourtant, on aimerait refouler ses sentiments, ne plus les laisser agir sur soi, ne plus penser à ce coup de tonnerre.

Cette maladie, cela peut être un cancer récemment diagnostiqué, un infarctus ou une hémorragie cérébrale, un grave accident, mais aussi une maladie chronique entravant les activités quotidiennes telles que la sclérose en plaques et certaines affections neurologiques, la polyarthrite chronique, le sida, mais aussi l'alcoolisme et les autres maladies de dépendance, la dépression ou la schizophrénie, les tentatives de suicide, les angoisses - la liste ne saurait être exhaustive.

\section{Dimanche 7 mars: journée des malades 2010}

Différentes études menées en Suisse et à l'étranger révèlent que les enfants réagissent fortement aux graves maladies de leur père ou leur mère. Selon leur âge et leur stade de développement, on peut, par exemple, noter ce qui suit:

- Chez les nourrissons, on constate des troubles de l'alimentation, de la digestion ou du sommeil induits par la peur d'une séparation ou d'une menace existentielle. Il est donc particulièrement important que le parent en bonne santé établisse une relation de confiance avec l'enfant.

- Les petits enfants peuvent développer des idées d'omnipotence leur faisant croire, par ex. qu'ils devraient être punis; ils se détachent alors de toute relation.

- Même à l'âge préscolaire, les enfants s'imaginent qu'ils pourraient - de façon magique - être la cause de la maladie ou être touchés par cette maladie.

- A l'âge scolaire, les enfants développent des peurs physiques. Ils estiment être une charge pour leurs parents et ont souvent des difficultés scolaires.

- A la puberté et un peu plus tard, les jeunes prennent mieux conscience de la maladie et de ses ef- fets; ils sont souvent affectés de peurs quant à son caractère héréditaire et sont tourmentés par leurs besoins d'autonomie et de délimitation par rapport au sentiment de responsabilité qu'ils ont à l'égard de leurs parents.

Outre le fait qu'il doit assumer son destin, le parent malade (et son partenaire) est souvent dépassé par la situation car il devrait encore trouver la force nécessaire pour comprendre les peurs de ses enfants et réagir correctement. Son incapacité à faire face est souvent ressentie comme un abandon par les enfants, ce qui peut encore aggraver les peurs existantes suite au refoulement de sentiments agressifs.

Le thème de la Journée des malades 2010 a pour but d'attirer l'attention sur les répercussions, souvent négligées, des maladies graves au sein de la famille. Une mesure préventive consiste à stabiliser les relations familiales. Médecins et soignants sont invités à approfondir leurs connaissances afin de savoir où l'on peut trouver de l'aide (si possible aussi pour le parent en bonne santé), compte tenu des troubles supposés ou constatés chez les enfants d'un parent gravement malade. Pour les petits enfants, il sera important de localiser des personnes de référence qui leur sont proches et, pour les enfants plus âgés, de les mettre en réseau avec d'autres personnes de référence (par ex. jardin d'enfants, école, services sociaux, paroisse), de manière à ce que l'enfant insécurisé mais ayant malgré tout besoin de s'informer et de parler, ne passe pas «à travers les mailles du filet». Selon la situation, l'intervention d'un psychiatre pour enfants et adolescents peut s'avérer nécessaire.

Il est particulièrement réjouissant de trouver dans le $2^{\mathrm{e}}$ cahier du Forum Médical Suisse un article sur l'offre thérapeutique en milieu hospitalier proposée en Thurgovie pour des petits enfants et leurs parents souffrant de maladies psychiques [1].

Pour terminer, voici la formule souhaitée par Dimitri pour la Journée des malades 2010 et qui reprend, en l'occurrence, la phrase célèbre du petit Prince d'Antoine de Saint-Exupéry «On ne voit bien qu'avec le cœur»: «Un sourire, une caresse, un geste d'amour, chaque enfant comprend cela. Sans le cœur, on ne voit pas bien.»

Vous trouverez de plus amples informations et renseignements sur les offres d'aide et de contact sur www.tagderkranken.ch.

u.steiner@hin.ch 